\title{
EVALUACIÓN DE LA ACTIVIDAD SINÉRGICA ENTRE QUITOSANO DE PLUMA DE POTA (DOSIDICUS GIGAS) Y ÁCIDO CÍTRICO EN EL PARDEAMIENTO DE LA PULPA DE CHIRIMOYA (Annona Cherimola MILL.)
}

\author{
Freddy Quispe ${ }^{* a}$, Yanina Villa ${ }^{\mathrm{a}}$, Noemí Bravo ${ }^{\mathrm{a}}$, Olivio Castro ${ }^{\mathrm{a}}$, \\ Julio Santiago ${ }^{\text {, }}$, Marco Guerrero ${ }^{a}$
}

\begin{abstract}
RESUMEN
El estudio de investigación consistió en tres etapas: primero obtener y caracterizar al quitosano extraído a partir de la pluma de pota, segundo seleccionar la variedad de chirimoya apropiada en función al rendimiento del fruto y tercero estudiar el efecto sinérgico entre el quitosano y ácido cítrico en el control del pardeamiento enzimático de la pulpa de chirimoya. En la caracterización del quitosano se obtuvo un valor de $846 \mathrm{kDa}$ de peso molecular y $78,2 \%$ del grado de desacetilación. Empleando un Diseño Completamente al Azar (DCA) se evaluó tres variedades de chirimoya (Lisa, Impresa y Umbonata) seleccionando la variedad Lisa por el mayor rendimiento en pulpa; luego se empleó un Diseño de Bloques Completamente al Azar (DBCA) evaluando cuatro tratamientos (T) por triplicado en la pulpa estandarizada, el cuarto tratamiento $(0,75 \%$ quitosano $+3 \%$ ácido cítrico) presentó la menor actividad de la polifenoloxidasa (70,80 unidades de $\mathrm{PPO} / \mathrm{min} \mathrm{x}$ g de muestra) y ralentizó la oxidación del contenido de polifenoles totales ( $28 \mathrm{mg}$ de ácido gálico/100 $\mathrm{g}$ en peso de pulpa fresca) durante la evaluación. La proyección de vida útil en la pulpa se obtuvo empleando la ecuación de Arrhenius y la variación de color, presentando el cuarto tratamiento el mayor tiempo (254 días), evidencia de un efecto sinérgico que contribuye a retrasar el pardeamiento enzimático en la pulpa de la chirimoya.
\end{abstract}

Palabras clave: Quitosano; chirimoya; pulpa; pardeamiento enzimático; efecto sinérgico.

\section{EVALUATION OF THE SYNERGISTIC ACTIVITY BETWEEN CHITOSAN FROM SQUID PLUME (DOSIDICUS GIGAS) AND CITRIC ACID IN THE BROWNING OF CUSTARD APPLE PULP (Annona cherimola Mill.)}

\begin{abstract}
The research study consisted of three stages: firstly, obtaining and characterizing the chitosan extracted from the squid plume, secondly selecting the appropriate variety of custard apple

a Facultad de Química e Ingeniería química, Universidad Nacional Mayor de San Marcos, Cercado de Lima 15081, Perú. freddy.quispe@unmsm.edu.pe.
\end{abstract}


based on the yield of the fruit, and thirdly, studying the synergistic effect between chitosan and citric acid on control of enzymatic browning of custard apple pulp. In the characterization of the chitosan a value of $846 \mathrm{kDa}$ of molecular weight and $78.2 \%$ of the degree of deacetylation was obtained. Using a Completely Randomized Design (CRD), three cherimoya varieties (Lisa, Impresa and Umbonata) were evaluated, selecting the Lisa variety for the highest pulp yield; then a Completely Randomized Block Design (CRBD) was used evaluating four treatments $(\mathrm{T})$ in triplicate in the standardized pulp, the fourth treatment $(0.75 \%$ chitosan + $3 \%$ citric acid) presented the lowest polyphenoloxidase activity (70,80 PPO units/ min xg of sample) and slowed the oxidation of the total polyphenol content $(28 \mathrm{mg}$ of gallic acid/100 $\mathrm{g}$ by weight of fresh pulp) during the evaluation. The projection of useful life in the pulp was obtained using the Arrhenius equation and the color variation, with the fourth treatment presenting the longest time (254 days), evidence of a synergistic effect that contributes to delaying the enzymatic browning in the pulp of the custard apple.

Keywords: chitosan; cherimoya; pulp; browning; synergistic effect.

\section{INTRODUCCIÓN}

El pardeamiento enzimático es la oxidación de compuestos fenólicos, catalizada principalmente por la enzima polifenoloxidasa (PPO), esta oxidación produce quinonas, las cuales se condensan y reaccionan rápidamente formando un conjunto de pigmentos oscuros indeseables (melaminas), causando una serie de cambios negativos en la calidad nutricional y sensorial, por ello, el pardeamiento enzimático ha sido preocupación importante en la industria y ciencia de los alimentos ${ }^{1}$.

Por otro lado, el quitosano es un producto natural, biodegradable y no tóxico, donde su principal característica es la capacidad de inducir mecanismos de resistencia en el fruto modificado, además se están realizando evaluaciones del efecto sinérgico entre el quitosano y otros productos de origen natural como posibles alternativas de control a los problemas de postcosecha y frutas procesadas ${ }^{2}$.

La chirimoya cultivada en Perú posee una alta aceptación en el mercado nacional e internacional debido a sus atributos sensoriales (sabor, textura, aroma, entre otros), sin embargo, el principal problema es el pardeamiento de la pulpa causado por la enzima polifenoloxidasa, que en la chirimoya, perteneciente al género Annona, solo se puede evitar con productos químicos, pues utilizar altas temperaturas provoca el desarrollo de sabores extraños, limitando la producción y posterior comercialización, por esta razón es considerado un cultivo de subsistencia a causa del bajo acceso a tecnología adecuada ${ }^{3}$, por consiguiente, se busca alternativas de conservación, con el fin de desarrollar tecnologías simples y económicas que retrasen los procesos de deterioro y mantengan la calidad e inocuidad microbiológica de los productos.

La industrialización de la pulpa de chirimoya logrará abrir nuevos mercados, tales como empresas de helados, pastelerías, entre otros, incrementando la demanda de este producto, 
reduciendo las pérdidas causadas por el deterioro de la fruta durante la manipulación y el transporte, asimismo al producir pulpa de chirimoya se obtiene un producto con valor agregado, por tanto, una alternativa de solución al problema socioeconómico presente en las zonas rurales productoras de este fruto.

El presente trabajo de investigación radica en buscar nuevas alternativas para la conservación de la pulpa de chirimoya evaluando su potencial efecto sinérgico entre el quitosano (CS) y el ácido cítrico $(\mathrm{AC})$, con la finalidad de ampliar la vida útil durante su almacenamiento en congelación, manteniendo las propiedades fisicoquímicas propias de la chirimoya fresca y a su vez conservar sus características sensoriales.

\section{PARTE EXPERIMENTAL}

El desarrollo del procedimiento experimental se llevó a cabo en el "Laboratorio de Investigación y Desarrollo de Química Orgánica” y el "Laboratorio de Síntesis Orgánica” del departamento de Química Orgánica correspondiente a la Facultad de Química e Ingeniería Química de la Universidad Nacional Mayor de San Marcos.

\section{Materia prima e insumos}

Chirimoya procedente del pueblo anexo La Merced de Chaute, distrito de San Bartolomé, provincia de Huarochirí, región Lima, ubicada a $2491 \mathrm{msnm}$.

Quitosano obtenido de la quitina extraída a partir del residuo pluma de pota recolectada en el Terminal Pesquero del distrito de Ventanilla (Callao - Perú).

Ácido cítrico (E330) - Procedente de la empresa peruana Marva S.A.C.

\section{Procedimiento experimental}

El presente trabajo de investigación comprende dos etapas:

Obtención del quitosano a partir de la pluma de pota (Figura 1). La pluma de pota recepcionada pasó por el proceso de lavado, picado, desproteinización utilizando $\mathrm{NaOH} \mathrm{10 \% ,} \mathrm{luego} \mathrm{fue}$ lavado consecutivamente hasta llegar a $\mathrm{pH}$ neutro para volver a ser atacado por $\mathrm{NaOH} 50 \%$ hasta lograr su desacetilación parcial del biopolímero, se lavó consecutivamente hasta llegar a $p \mathrm{H}$ neutro, finalmente fue llevado a estufa hasta su secado, siendo almacenado en un envase de vidrio. 


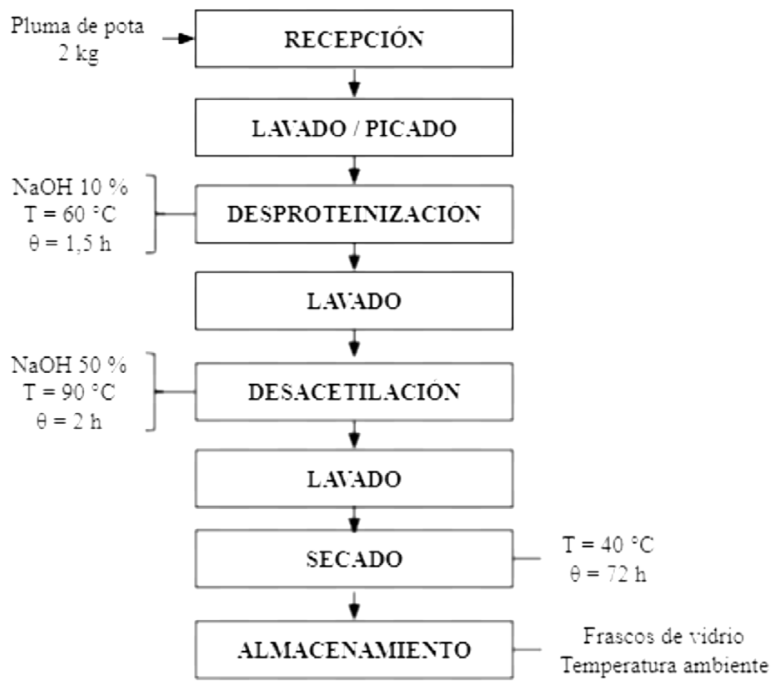

Figura 1. Proceso de extracción y obtención del quitosano a partir de la pluma de pota.

La obtención de la pulpa de chirimoya (Figura 2). Las chirimoyas se recepcionaron en el laboratorio, donde se seleccionaron las frutas maduras, sanas y de tamaño uniformes, luego se lavaron y desinfectaron, posteriormente se realizó el pelado, pulpeado y estandarizado con los diferentes tratamientos de quitosano y ácido cítrico. Las soluciones formadas fueron agitadas durante a $800 \mathrm{rpm}, 70^{\circ} \mathrm{C}$ durante $4 \mathrm{~h}$. Se formaron 4 soluciones:

- Tratamiento control o blanco (T1): Agua destilada

- Tratamiento 2 (T2): Solución de ácido cítrico al 3\%

- Tratamiento 3 (T3): Solución de quitosano al 0,75\%

- Tratamiento 4 (T4): Solución de quitosano al 0,75\% y ácido cítrico al 3\%

Las diferentes soluciones se añadieron a la pulpa de chirimoya en relación de 1/27 respectivamente. Las muestras de pulpas estandarizadas se envasaron y almacenaron en congelación hasta su posterior análisis. 


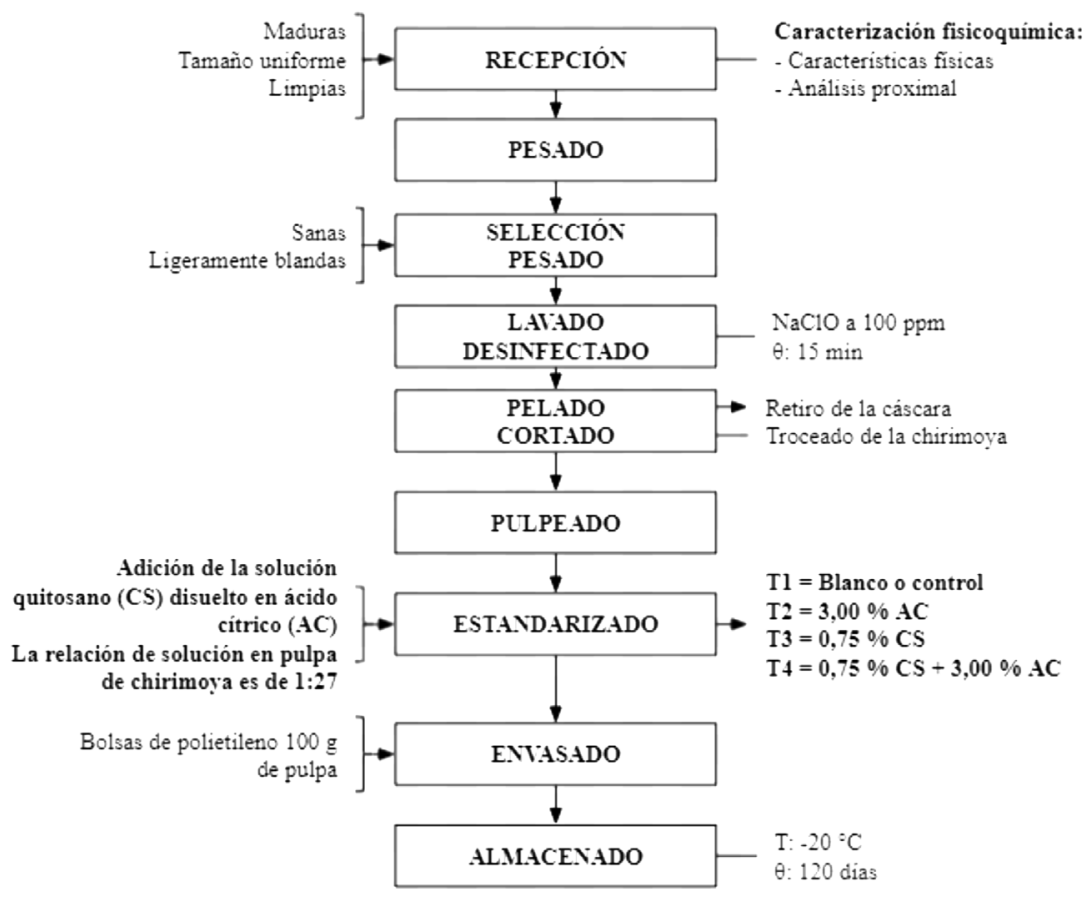

Figura 2. Flujograma para la obtención, envasado y almacenamiento de la pulpa de chirimoya.

\section{Caracterización del quitosano obtenido}

Humedad. Se colocaron $1,5 \mathrm{~g}$ de quitosano en estufa durante $48 \mathrm{~h}$ a $70{ }^{\circ} \mathrm{C}$ hasta obtener un peso constante. El porcentaje de humedad se obtiene a partir de la siguiente ecuación 1:

$$
\% \text { Humedad }=\frac{\text { Peso inicial }- \text { Peso final }}{\text { Peso muestra de quitosano }} \times 100 \%
$$

Cenizas. Se llevó a mufla $1,5 \mathrm{~g}$ de quitosano durante $8 \mathrm{~h}$ a $500{ }^{\circ} \mathrm{C}$, luego se dejó enfriar en un desecador hasta llegar a temperatura ambiente. El porcentaje de cenizas se obtuvo a partir de la siguiente ecuación 2:

$$
\% \text { Cenizas }=\frac{\text { Peso del crisol }+ \text { cenizas }- \text { Peso del crisol vacío }}{\text { Peso de la muestra seca }} \times 100 \%
$$

Grado de desacetilación. Se mezclaron $2 \mathrm{mg}$ de quitosano con $98 \mathrm{mg}$ de $\mathrm{KBr}$, colocando la mezcla en el pastillador durante $30 \mathrm{~min}$, por último, se colocó la pastilla formada en el equipo IR, mediante el software del equipo se obtuvo el espectro de la muestra, reportando las absorbancias para la frecuencia de $1320 \mathrm{~cm}^{-1}$ y $1420 \mathrm{~cm}^{-1}$. El grado de desacetilación se calculó aplicando la siguiente ecuación 3: 


$$
\% \mathrm{GD}=87,8-31,92 \frac{\mathrm{A}_{1320}}{\mathrm{~A}_{1420}}
$$

Peso molecular. Mediante la ecuación 4 de Mark-Houwink se determinó el peso molecular del quitosano ${ }^{4}$.

$$
[\eta]=K(\bar{M})^{\alpha}
$$

Donde:

$\eta$ : Viscosidad intrínseca $(\mathrm{ml} / \mathrm{g})$.

$\mathrm{K}, \alpha$ : Constantes que varían según el grado de desacetilación del polímero.

M: Peso molecular (KDa).

Empleando las ecuaciones 5 y 6 se halló los valores de las constantes $\mathrm{K}$ y $\alpha$ en función del porcentaje del grado de desacetilación ${ }^{4}$.

$$
\begin{aligned}
& \mathrm{k}=1,64 \times 10^{-30} \times(\% \mathrm{GD})^{14} \ldots \ldots . \\
& \alpha=1,82-1,02 \times 10^{-2} \times(\% \mathrm{GD})
\end{aligned}
$$

\section{Selección de la variedad de chirimoya}

Rendimiento del fruto. Con el fin de determinar la proporción de cáscara, semillas y pulpa se separó las tres partes mencionadas para ser pesadas en una balanza de mesa, obteniendo el porcentaje de pepa, cáscara y pulpa para cada variedad.

\section{Estudio de la actividad sinérgica}

Contenido de polifenoles totales. El contenido total de polifenoles se determinó aplicando el método de Folin Ciocalteu basado en la descripción de García et al. ${ }^{5}$ con algunas modificaciones. Se pesó $2,5 \mathrm{~g}$ de pulpa de chirimoya y se añadió $5 \mathrm{ml}$ de etanol puro, homogeneizando el contenido mediante agitación constante durante $2 \mathrm{~min}$, luego se filtró el contenido con tela de organza recuperando el líquido sobrenadante, el cual contiene los compuestos polifenólicos presentes en la pulpa de chirimoya. En cada vial se añadió 3,9 $\mathrm{ml}$ de agua destilada, agregando $50 \mu \mathrm{L}$ de la extracción de los compuestos polifenólicos o de cada disolución patrón de ácido gálico, en seguida, se añadió $250 \mu \mathrm{L}$ del reactivo Folin Ciocalteu, dejando reposar todo el contenido durante $8 \mathrm{~min}$ en oscuridad. Transcurrido el tiempo, se le adicionó $750 \mu \mathrm{L}$ una solución de $\mathrm{Na}_{2} \mathrm{CO}_{3}$ al 7,5\%, dejándolo reposar durante $2,5 \mathrm{~h}$ a temperatura ambiente y en oscuridad. Finalmente, la lectura del contenido total de polifenoles se cuantificó midiendo su absorbancia a una longitud de onda de $765 \mathrm{~nm}$.

Actividad de la Polifenoloxidasa. Se utilizó el método espectrofotométrico, pesando 2,5 g de pulpa y homogeneizando con $10 \mathrm{ml}$ de buffer McIlvaine $(p \mathrm{H} 7,0)$ durante $30 \mathrm{~s}$, luego se filtró desechando el residuo sólido, finalmente la solución filtrada se centrifugó a 2000 rpm durante $10 \mathrm{~min}$, siendo el líquido sobrenadante el extracto que contiene la enzima polifenoloxidasa. 
Se utilizó como sustrato al pirocatecol, en una cubeta se añadió $2,5 \mathrm{ml}$ de pirocatecol a 0,01 M y $100 \mu \mathrm{L}$ del extracto enzimático, como blanco se tomó $2,5 \mathrm{ml}$ de pirocatecol y $100 \mu \mathrm{L}$ del buffer McIlvaine ( $p \mathrm{H} 7,0)$. Se realizaron lecturas de absorbancia a $400 \mathrm{~nm}$ cada 10 s durante 2 min en un espectrofotómetro UV - Vis. Una unidad de la enzima polifenoloxidasa, se define como la cantidad de extracto enzimático que causa un aumento en la absorbancia de 0,001 unidades por minuto.

Variación del color. La variación del color $(\Delta \mathrm{E})$ producido en la pulpa de chirimoya durante los 120 días de almacenamiento se registró utilizando el sistema CIE-Lab, donde se midió los parámetros L*, a* y b*. El parámetro L* nos muestra valores de luminosidad u oscuridad, mientras que el parámetro a* nos indica la variación del color rojo - verde, siendo positivo (+a) para el color rojo y negativo (-a) el color verde, mientras que los valores del parámetro b nos explica la variación del color amarillo - azul, donde valores positivos $(+b)$ indica el color amarillo y valores negativos el color azul ${ }^{6}$. La variación del color $(\Delta \mathrm{E})$ se obtuvo luego de aplicar la ecuación 7, especificada en la norma europea EN 12878:

$$
\Delta \mathrm{E}=\sqrt{\Delta \mathrm{L}^{* 2}+\Delta \mathrm{a}^{* 2}+\Delta \mathrm{b}^{* 2}}
$$

\section{Análisis de la información}

Durante el proceso de evaluación de la actividad sinérgica se recopilaron cada uno de los datos por triplicado, los cuales fueron mostrados como el promedio \pm la desviación estándar empleando Hoja de Cálculo de Google Drive. Para el tratamiento estadístico en la selección de la variedad de chirimoya se utilizó un Diseño Completamente al Azar (DCA), en tanto para la evaluación de la actividad sinérgica presente en la pulpa de chirimoya, el Diseño de Bloques Completamente al Azar (DBCA) con un arreglo factorial 2x2, cuyos datos fueron analizados mediante el software estadístico InfoStat versión 2019 realizando el análisis de varianza de los factores (ANOVA) con un nivel de significancia de 0,05 aplicando la prueba de comparaciones múltiples de Tukey para determinar si existe diferencia significativa entre los tratamientos evaluados.

\section{RESULTADOS Y DISCUSIÓN}

\section{Caracterización del quitosano}

Debido a la importancia de los grupos aminas presente en la estructura química del quitosano, se realizó la caracterización de este biopolímero tomando en cuenta su porcentaje de humedad, contenido de cenizas, peso molecular y el grado de desacetilación, dichos resultados se detallan en la Tabla 1 .

Tabla 1. Caracterización del quitosano.

\begin{tabular}{cccc}
\hline Humedad (\%) & Cenizas (\%) & Peso molecular (KDa) & Grado de desacetilación (\%) \\
\hline 10,52 & 0,35 & 846 & 78,2 \\
\hline
\end{tabular}


Experimentalmente se determinó un valor de $0,35 \%$ de cenizas para el quitosano extraído de la quitina presente en la pluma de pota (Dosidicus gigas), siendo este un resultado muy bajo a comparación de los reportados por otros autores en la caracterización del quitosano proveniente de distintas fuentes de materia prima. Posiblemente, el bajo contenido de cenizas, se deba a las características y cualidades que presenta la quitina obtenida a partir del cartílago (pluma) del calamar gigante o pota, cuya fuente carece de material calcáreo y se ubica en el interior del cefalópodo, por lo que, no se lleva a cabo una bioacumulación directa de metales inorgánicos como el plomo $(\mathrm{Pb})$, mercurio $(\mathrm{Hg})$, arsénico $(\mathrm{As})$, cadmio $(\mathrm{Cd})$, cromo (Cr), Zinc (Zn) y Níquel (Ni) a diferencia de la quitina que se encuentra en el exoesqueleto de moluscos y crustáceos, donde la exposición con estos metales pesados, producto de la contaminación del mar peruano, ocurre de forma directa logrando obtener porcentajes de cenizas elevadas $(>1 \%)$, generando un gran impacto a la salud debido a su alta toxicidad de estos materiales inorgánicos ${ }^{4,7}$.

El contenido de humedad determinado fue de 10,52\%, cuyo valor es menor comparado con el $14 \%$ de humedad que reportaron para el quitosano extraído a partir de la quitina de camarón8. Los diferentes contenidos de humedad que presentan los compuestos de quitosano se generan, principalmente, por las distintas fuentes de materias primas de obtención, además de los procesos físicos o de reacción bruscos al cual se somete la quitina durante el proceso de desacetilación, disminuyendo su contenido de agua, obteniendo polímeros con diferentes propiedades y características?.

El espectro infrarrojo (IR) obtenido para la muestra de quitosano se detalla en la Figura 3, cuyas bandas de absorción se obtuvieron luego de la interacción entre la energía producida por el espectro infrarrojo y la muestra de quitosano, estas fueron de 3414, 2889, 1433, 1632, $1382,1321,1255$ y $1083 \mathrm{~cm}^{-1}$.

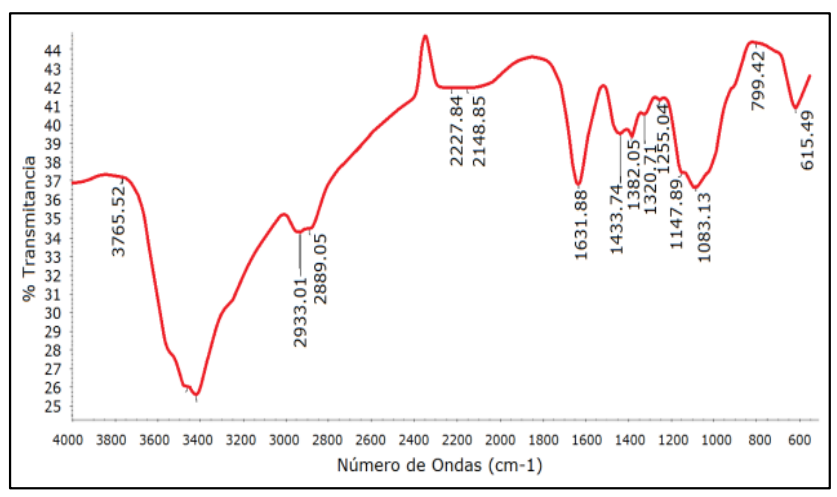

Figura 3. Espectro infrarrojo (IR) de la muestra de quitosano. 
El pico formado alrededor de 1321 y $1433 \mathrm{~cm}^{-1}$ se le atribuye a la vibración de flexión del enlace $\mathrm{C}$ - N presente en el grupo amida y amina respectivamente, las cuales son utilizadas como bandas de medición logrando determinar el porcentaje del grado de desacetilación debido a la posibilidad de hallar la relación que existe entre la cantidad de N-glucosamina y $\mathrm{N}$-acetilglucosamina en el quitosano ${ }^{4}$. Aplicando la espectroscopía FTIR, el grado de desacetilación para el quitosano obtenido a partir de la pluma de pota fue de $78,2 \%$, cuyo valor se encuentra por debajo de $85,47 \%$ del GD reportado por Moreno et al. ${ }^{10}$, a pesar que el quitosano proviene de la misma fuente de obtención (pluma de pota). Probablemente, esta diferencia de valores, comparando con nuestros resultados, se deba al cálculo de las áreas debajo de las bandas de absorción en 1321 y $1433 \mathrm{~cm}^{-1}$, las cuales se tomaron como referencia siguiendo la metodología propuesta por $\mathrm{Castro}^{4}$.

La obtención del grado de desacetilación es una propiedad determinante en la clasificación del polímero como quitina o quitosano, siendo considerado como quitina si presenta un valor menor del $50 \%$ del grado de desacetilación ${ }^{4}$. Por los resultados obtenidos (78,2\% del GD) y los grupos funcionales reconocidos en el espectro IR, se puede afirmar que el biopolímero obtenido a partir de la pluma de pota es clasificado como quitosano.

Otras de las evaluaciones muy recurrentes en la caracterización del quitosano es la determinación de su peso molecular, experimentalmente se obtuvo un peso molecular de $846 \mathrm{KDa}$, este resultado es menor comparado a lo obtenido por Moreno et al. ${ }^{10}$, quienes determinaron un peso molecular de $1055 \mathrm{KDa}$ del quitosano proveniente de la misma materia prima.

\section{Selección de la variedad de chirimoya}

En la Tabla 2 se indican los rendimientos (en porcentaje) para las diferentes variedades de la chirimoya. Siendo la variedad Lisa, la de menor rendimiento en cáscara $(26,31 \%)$ y pepa $(3,10 \%)$ a diferencia de las otras dos variedades (Impresa y Umbonata). Sin embargo, el rendimiento en pulpa de la variedad Lisa fue de 70,07\%, resultado mayor a comparación de la variedad Impresa $(63,74 \%)$ y Umbonata $(66,37 \%)$.

Tabla 2. Valores promedio del rendimiento de cáscara, pepa y pulpa para las tres variedades de chirimoya.

\begin{tabular}{cccc}
\hline Variedad & $\begin{array}{c}\text { Rendimiento de cáscara } \\
(\mathbf{\%})\end{array}$ & $\begin{array}{c}\text { Rendimiento de } \\
\text { pepa (\%) }\end{array}$ & $\begin{array}{c}\text { Rendimiento de } \\
\text { pulpa (\%) }\end{array}$ \\
\hline Lisa & $26,31^{\mathrm{A}} \pm 1,33$ & $3,10^{\mathrm{A}} \pm 0,22$ & $70,07^{\mathrm{A}} \pm 2,01$ \\
Impresa & $28,65^{\mathrm{B}} \pm 0,77$ & $7,16^{\mathrm{C}} \pm 0,41$ & $63,74^{\mathrm{B}} \pm 2,96$ \\
Umbonata & $29,23^{\mathrm{B}} \pm 1,34$ & $6,26^{\mathrm{B}} \pm 0,49$ & $66,37^{\mathrm{AB}} \pm 2,23$
\end{tabular}

Nota. Los valores luego del signo \pm indican la desviación estándar. Las letras A, B o AB, situadas como superíndice en las medias, son una simbología práctica, donde la letra común no es significativamente diferente. 
De los diferentes análisis estadísticos, se deduce que la chirimoya variedad Lisa es apropiada para procesar a nivel industrial, esto debido a los resultados en rendimiento de cáscara, pepa y pulpa, evidenciando el mayor aprovechamiento de la fruta a comparación de las otras dos variedades.

\section{Estudio de la actividad sinérgica entre quitosano y ácido cítrico en la pulpa de chirimoya}

Con la finalidad de observar una posible acción sinérgica del quitosano junto al ácido cítrico para controlar el pardeamiento enzimático en la pulpa de chirimoya, se evaluó la variación de los compuestos fenólicos durante 120 días de almacenamiento a $-20^{\circ} \mathrm{C}$, permitiendo estudiar la posible interdependencia entre el contenido de polifenoles totales y el desarrollo de este fenómeno enzimático. La Figura 4 muestra la variación en contenido de polifenoles totales para cada tratamiento estudiado, los resultados se expresan en mg de ácido gálico/100g de pulpa de chirimoya.

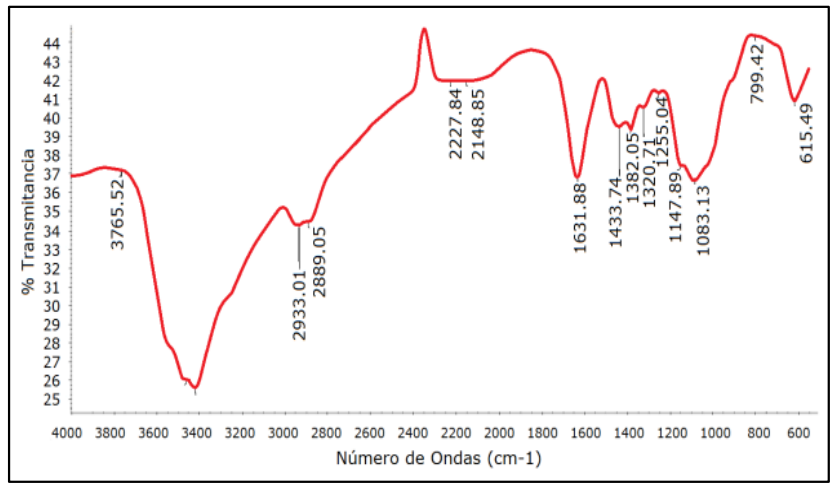

Figura 4. Variación del contenido de polifenoles totales con respecto a los días de almacenamiento de la pulpa de chirimoya.

Durante los 120 días de evaluación, los cuatro tratamientos mostraron una caída de los compuestos polifenólicos, esto probablemente se deba a la degradación oxidativa que sufre estos compuestos al reaccionar con la enzima polifenoloxidasa en presencia del oxígeno provocando el pardeamiento enzimático en la pulpa ${ }^{11}$.

El tratamiento control o blanco (T1) presentó la mayor variación de estos compuestos, registrando una oxidación total de 49,4 mg de ácido gálico/100 g de muestra, sin embargo, el tratamiento $\mathrm{T}_{4}$, cuya solución está formada por la mezcla de quitosano y ácido cítrico, mostró un comportamiento antagónico con una disminución total de $28 \mathrm{mg}$ de ácido gálico/100 g de pulpa, siendo este el tratamiento que presentó menor variación, asimismo los tratamientos $\mathrm{T}_{2}$ y $\mathrm{T}_{3}$ reportaron oxidaciones enzimáticas de 33,8 y 36,17 mg de ácido gálico/100 g de muestra respectivamente. La composición polifenólica que presenta la chirimoya es de gran relevancia en la comprensión de la rapidez del pardeamiento que sufre la pulpa, además en base a las investigaciones realizadas por Barea ${ }^{12}$, se afirma que la chirimoya presenta, 
en su perfil polifenólico, cantidades notables de derivados del ácido hidroxicinámico (ácido cafeico, ácido ferúlico y el ácido cumárico), flavonoides y taninos, los cuales son degradados mediante oxidación enzimática produciendo compuestos muy reactivos llamadas quinonas, que al condensarse forman polímeros pardos conocidos como melaminas; por esta razón, el tratamiento control $\left(\mathrm{T}_{1}\right)$ al no presentar algún agente antioxidante que ayude a controlar la oxidación enzimática, muestra un rápido pardeamiento debido a la alta velocidad de oxidación de los polifenoles presentes en la pulpa, caso contrario a los resultados obtenidos por los tratamientos $\mathrm{T}_{4} \mathrm{y} \mathrm{T}_{3}$, formulados con soluciones de quitosano, debido a la capacidad antioxidante que presenta las soluciones de este biopolímero actuando como agente antioxidante secundario por su eficiencia de quelación contra iones metálicos, los cuales ralentizan el inicio de la oxidación ${ }^{13}$. La propiedad quelante, del quitosano en diferentes alimentos, se debe a la interacción entre el quitosano y iones metálicos de transición y postransición, como por ejemplo el $\mathrm{Cu}^{+2}$, que forma parte del grupo prostético de la polifenoloxidasa dando inicio al pardeamiento enzimático; esta interacción se logra gracias a los grupos aminas e hidroxilos que presenta el quitosano en su estructura química, comportándose como un polielectrolito catiónico, que por debajo de un $\mathrm{pH}$ 6,5 presenta una alta densidad de carga formando quelatos con los iones metálicos ${ }^{14}$.

Además, un $p \mathrm{H}$ bajo puede retardar el crecimiento de microorganismos como las bacterias, así también, inactivar enzimas no deseables como la polifenoloxidasa, por esta razón, el ácido cítrico también mostró un efecto significativo con respecto a la variación de los polifenoles totales presentes en la pulpa de chirimoya, debido a su baja actividad por debajo de un $p \mathrm{H}$ 4 , constituyendo una forma de control del pardeamiento enzimático ${ }^{15}$. La reducción de la actividad de la PPO provoca una disminución en las reacciones del pardeamiento enzimático, en consecuencia, la oxidación de compuestos polifenólicos a orto-quinonas se vería también reducido.

En la Figura 5 se observa las actividades de la PPO generadas por los tratamientos en estudio, donde $\mathrm{T}_{4}$ presentó la menor actividad enzimática durante toda la evaluación, seguido por los tratamientos $\mathrm{T}_{2} \mathrm{y} \mathrm{T}_{3}$, sin embargo, el tratamiento control $\left(\mathrm{T}_{1}\right)$ presentó la mayor actividad. Según Jiang et al. ${ }^{16}$ afirman que una elevada actividad enzimática de la polifenoloxidasa afecta en la rapidez del pardeamiento de frutas que presentan concentraciones importantes de compuestos polifenólicos como es el caso de la chirimoya, entonces el tratamiento $\mathrm{T}_{1}$, al poseer una elevada actividad enzimática a comparación del resto de tratamientos, presenta un rápido pardeamiento a causa de la mayor concentración de polímeros pardos producidos, este resultado se encuentra altamente relacionado con los cambios de color que se produce en la pulpa de la chirimoya y la rápida oxidación de sus polifenoles, mostrados en la Figura 4, generando indeseables cambios sensoriales y nutricionales ${ }^{3}$. 


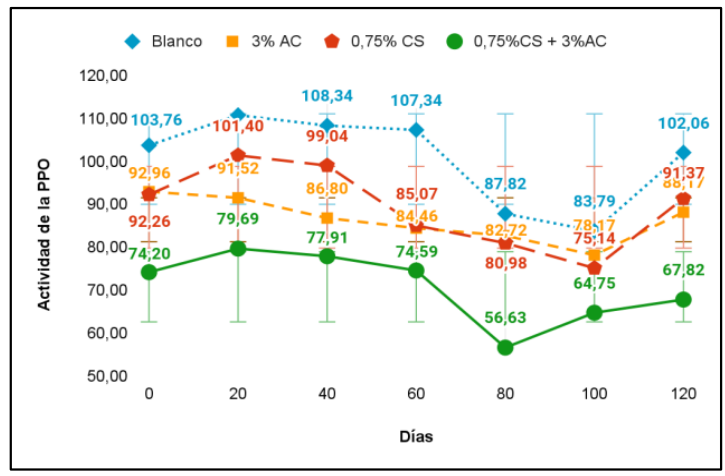

Figura 5. Variación de la actividad de la polifenoloxidasa con respecto a los días de almacenamiento de la pulpa de chirimoya.

La elevada actividad de la PPO, hallada experimentalmente, se puede deber al desorden celular que se genera al recibir daños mecánicos propios del pulpeado, esta explicación se ve reforzada por Morante et al. ${ }^{11}$, donde mencionan que los tejidos vegetales sin lesión e impacto mecánico, se encuentran en compartimentos diferentes, los sustratos polifenólicos en la vacuola y la enzima polifenoloxidasa en el cloroplasto, evitando una reacción oxidativa, sin embargo, procesamientos industriales como el triturado, cortado o pulpeado aplicados en frutas, puede generar desorganizaciones celulares de estos compartimientos, permitiendo un contacto directo entre la enzima y los mismos compuestos fenólicos.

Los resultados muestran a la solución formulada por la combinación de quitosano $(0,75 \%)$ con ácido cítrico $(3,0 \%)$ como mejor tratamiento, evidenciando algún modo de interacción o entrecruzamiento entre ambos compuestos que ayudan a potencializar sus propiedades antioxidantes y quelantes obteniendo mejores resultados de inhibición y control del pardeamiento enzimático en la pulpa de chirimoya a comparación de emplear soluciones de quitosano o ácido cítrico independientemente ${ }^{17}$.

La disminución significativa en la actividad de la PPO tras aplicar una solución de ácido cítrico al $3 \%$, tratamientos $\mathrm{T}_{2} \mathrm{y} \mathrm{T}_{4}$, se puede deber a su capacidad acidulante generando ciertos cambios en el $p \mathrm{H}$ de la pulpa, por otro lado, Cob et al. ${ }^{18}$ también indican el efecto que produce la variación del $p \mathrm{H}$ respecto a la disminución de la actividad enzimática, siendo la actividad reducida a valores de $p \mathrm{H}$ alrededor de 4 . Respecto a la baja actividad hallada en la pulpa de chirimoya, para los tratamientos que presentan soluciones de quitosano como parte de su formulación $\left(\mathrm{T}_{3}, \mathrm{~T}_{4}\right)$, probablemente se explique por la interacción entre los grupos amina e hidroxilo reactivos del polímero con iones metálicos como el $\mathrm{Cu}^{+2}$, esta capacidad quelante del quitosano se ve afectada por la variación del $\mathrm{pH}$ modificando la densidad de

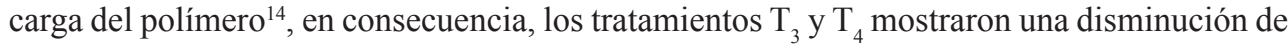
la actividad enzimática, esto posiblemente a los quelatos formados producto de la interacción 
entre el biopolímero y el cobre. A pesar de las diversas propiedades que brinda el quitosano y el ácido cítrico en el control del pardeamiento, existen investigaciones donde se estudia un posible entrecruzamiento entre ambos compuestos, Chávez et al. ${ }^{17}$ mencionan que esta reacción se puede dar mediante una esterificación o amidación. El entrecruzamiento por esterificación se obtiene mediante un enlace entre los grupos hidroxilos del quitosano y ácido cítrico, mientras que la reacción de amidación se logra mediante un enlace producido entre el grupo amino del quitosano con el grupo carboxílico del ácido cítrico formando un enlace amida luego de recibir calor. El tratamiento $\mathrm{T}_{4}$, cuya formulación esta compuesta por una solución de quitosano y ácido cítrico, fue agitada durante $4 \mathrm{~h}$ a $70^{\circ} \mathrm{C}$ produciendo, a esta temperatura, un entrecruzamiento entre estos dos compuestos mediante enlaces amida ${ }^{19}$.

Respecto a la cuantificación de los cambios de color $(\Delta \mathrm{E})$ producidos en la pulpa de chirimoya durante su almacenamiento, se registraron los valores para los cuatro tratamientos de estudio, donde se calculó en base a la ecuación especificada por la norma europea EN 12878 tomando en cuenta el sistema CIE-lab ${ }^{6}$.

De la Figura 6 se observa que todos los tratamientos presentaron un similar comportamiento respecto a los cambios del color producidos en la pulpa de chirimoya obteniendo rectas de pendientes positivas cuyas correlaciones lineales fueron adecuadas.

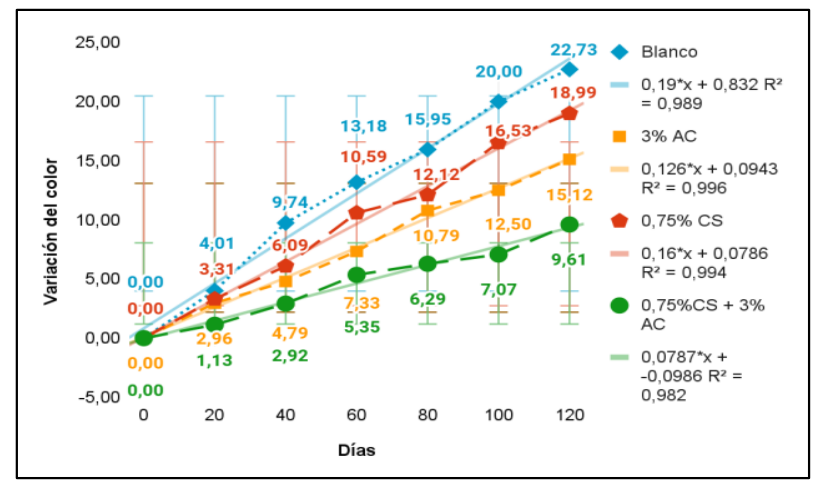

Figura 6. Variación de la actividad de la polifenoloxidasa con respecto a los días de almacenamiento de la pulpa de chirimoya.

El tratamiento $\mathrm{T}_{4}$ presentó una correlación lineal de $\mathrm{R}^{2}=0,982$ obteniendo los menores cambios de color durante toda la evaluación, seguido por lo tratamientos donde se involucra agentes antioxidantes de forma independiente, siendo el $\mathrm{T}_{3}\left(\mathrm{R}^{2}=0,994\right)$ quien presentó una recta más pronunciada a comparación del $\mathrm{T}_{2}\left(\mathrm{R}^{2}=0,996\right)$, esto nos indica que existe una mayor variación del color en la pulpa con solución de quitosano $(0,75 \%)$ en comparación a la tratada con ácido cítrico $(3 \%)$, mientras el tratamiento control $\left(\mathrm{T}_{1}\right)$ con un $\mathrm{R}^{2}=0,989$ registró el mayor cambio respecto al color inicial de la pulpa. 
El tratamiento control $T_{1}$ mostró la mayor diferencia total de 22,73 para el día 120 , probablemente el rápido cambio de color se encuentra relacionado con la alta actividad de la polifenoloxidasa registrada para la pulpa de chirimoya que no presenta la adición de algún agente antipardeante, de forma antagónica el tratamiento cuya formulación presenta la mezcla entre la solución de quitosano y ácido cítrico $\left(\mathrm{T}_{4}\right)$ mostró la menor variación total del color $(\Delta \mathrm{E})$, siendo esta diferencia de tan solo un 9,61 para el último día de evaluación. El efecto sinérgico entre ambos compuestos de estudio, generó una disminución en la actividad de la polifenoloxidasa, en consecuencia una lenta degradación oxidativa de los compuestos fenólicos de la fruta, esto evidenciaría un menor pardeamiento enzimático de la pulpa constatando la estrecha relación entre el cambio del color y el oscurecimiento enzimático oxidativo $^{11}$, entre tanto, los tratamientos $\mathrm{T}_{2} \mathrm{y} \mathrm{T}_{3}$, en donde se involucra la adición de soluciones independientes de ácido cítrico y quitosano respectivamente, consiguieron menores cambios del color a comparación del control (T1), pero mayor a la variación obtenida por el $\mathrm{T}_{4}$, siendo esta diferencia total de 15,12 y 18,99 para la pulpa tratada con solución de ácido cítrico y quitosano respectivamente.

Ante la actual problemática sobre la oxidación enzimática de la chirimoya, causada por la polifenoloxidasa, cuando la fruta es procesada, además de los escasos trabajos de investigación realizados para contrarrestar el fenómeno de oscurecimiento en pulpa de chirimoya, se procedió a realizar un análisis de la cinética química, donde se observa estudios relacionados con la diferencia del color en diversas frutas debido a su relación con la pérdida de la calidad sensorial; la investigación realizado por Ibarz et al. ${ }^{20}$ dejan abierta la posibilidad de aplicar la ecuación de Arrhenius en curvas o rectas que presenten un modelo cinético de primer orden u orden cero, al evaluar la rapidez del pardeamiento no enzimático a diferentes temperaturas encontraron un alto nivel de confianza (99\%) con una variación lineal del color en zumos de frutas. Ante la similitud de los resultados y la elevada correlación lineal para cada una de los tratamientos, existe la probabilidad de pronosticar el tiempo de vida útil en función a los cambios de color que se produce en la pulpa durante el almacenamiento, siendo este valor un parámetro importante por ser considerado un indicador de pérdida de la calidad sensorial. La Figura 7 muestra imágenes del avance del oscurecimiento oxidativo durante las fechas de evaluación, siendo el tratamiento control el que mostró a los 100 días de almacenamiento un cambio de color $(\Delta \mathrm{E}=20)$ en el que se consideró un pardeamiento de la pulpa, perceptible por el ojo humano, con probabilidad de rechazo por parte del consumidor. 


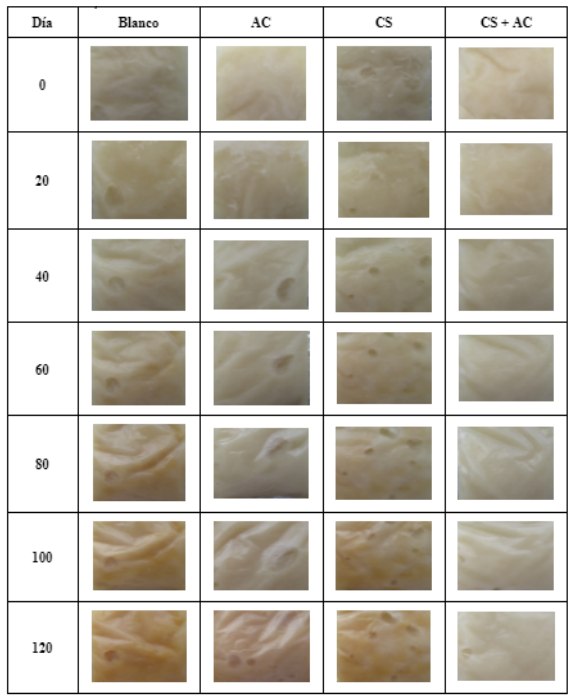

Figura 7. Cambio del color producto del pardeamiento enzimático de la pulpa de chirimoya.

En base a la ecuación de Arrhenius se muestra el siguiente modelo: $\Delta \mathrm{E}=\Delta \mathrm{E}^{*}+\mathrm{KT}$ el cual obedece a la regla de correspondencia de la variación del color (Figura 6). Para lograr estimar la vida útil de los diferentes tratamientos se tomó, como índice final de calidad, la diferencia del color conseguido por el tratamiento control hasta el día $100(\Delta \mathrm{E}=20)$, este valor fue considerado como referencia debido a la evidencia del pardeamiento en la pulpa, de tal modo, el tratamiento $\left(\mathrm{T}_{3}\right)$ obtuvo una vida útil de 125 días según el modelo cinético que lo rige $(\Delta \mathrm{E}=0,16+0,0786 \mathrm{~T})$, mientras el $\mathrm{T}_{2}$, una vida útil de 158 días según la ecuación que rige $(\Delta \mathrm{E}=0,126+0,0943 \mathrm{~T})$, sin embargo, el tratamiento $\mathrm{T}_{4}$, con un modelo cinético de $\Delta \mathrm{E}=$ $0,0787+0,0986 \mathrm{~T}$, lograría un tiempo de vida aproximado de 254 días en almacenamiento $\left(-20^{\circ} \mathrm{C}\right)$, Concluyendo, en base a todos los resultados mostrados, que el tratamiento $\mathrm{T}_{4}$ retrasó el pardeamiento enzimático en la pulpa de chirimoya.

\section{CONCLUSIONES}

El quitosano, extraído a partir de la pluma de pota, empleada en la presente investigación ostenta las siguientes características fisicoquímicas: 10,52\% de humedad, $0,35 \%$ de cenizas, $846 \mathrm{kDa}$ de peso molecular y 78,2\% del grado de desacetilación.

La chirimoya de variedad Lisa es la mejor, debido a que obtuvo un 70,07\%; 3,10\% y 26,31\% en rendimiento adecuado de pulpa, pepa y cáscara respectivamente. 
El tratamiento cuatro $\left(\mathrm{T}_{4}\right)$ cuya formulación presenta quitosano al $0,75 \%$ y ácido cítrico al $3 \%$ es el mejor, porque presentó la menor variación en el contenido de polifenoles totales, actividad de la enzima polifenoloxidasa y cambios de color, registrando valores de $28 \mathrm{mg}$ de ácido gálico/100 g de pulpa en fresco, 70,80 Unidades de PPO/min x g de muestra y 9,61 de variación del color respectivamente. Los estudios cinéticos proyectan un tiempo de vida útil, para el tratamiento $\mathrm{T} 4$, hasta 254 días en almacenamiento a $-20^{\circ} \mathrm{C}$.

\section{AGRADECIMIENTOS}

A nuestra alma mater, la Universidad Nacional Mayor de San Marcos "Decana de América", por el prestigio y la calidad de enseñanza, en especial a nuestra querida E.P. de Ingeniería Agroindustrial, perteneciente a la Facultad de Química e Ingeniería Química, por ser el lugar donde recibimos los conocimientos necesarios para formarnos como profesionales. A la Química Marlene Velásquez Yucra y los profesores e investigadores Jose Francisco Román Ferreyra y José Manuel Churata Chávez, por las recomendaciones durante las evaluaciones fisicoquímicas en las pruebas preliminares, siendo de gran ayuda para la obtención de resultados correctos.

\section{REFERENCIAS BIBLIOGRÁFICAS}

1. Guerrero CA. Inhibición de la actividad enzimática de la polifenoloxidasa extraída del banano (Cavendish valery) mediante sistemas bifásicos acuosos con isoespintanol y ácido ascórbico. [tesis de maestría]. Medellín: Universidad Nacional de Colombia, Sede Medellín, Facultad de Ciencias Agropecuarias, Departamento de Ingeniería Agrícola y de Alimentos; 2009.

2. Bautista S, Hernández AN, Velázquez MG, Bosquez E, Sánchez D. Quitosano: una alternativa natural para reducir microorganismos postcosecha y mantener la vida de anaquel de productos hortofrutícolas. Revista Iberoamericana De Tecnología Postcosecha. 2015; 7 (1): 1-6.

3. Kobashigawa SS. Análisis de oportunidades comerciales en mercados exigentes de la chirimoya a partir del desarrollo de la cadena productiva en Huaura. [tesis de pregrado]. Lima: Universidad Nacional Agraria La Molina.

4. Castro O. Investigación aplicada con quitina y quitosano. Lima, Perú: Editorial Académica Española; 2017.

5. García EM, Fernández I, Fuentes A. Determinación de polifenoles totales por el método Folin-Ciocalteu. Valencia: Universidad Politécnica de Valencia, Departamento de Tecnología de Alimentos; 2015.

6. López A, Di Sarli AR. El modelo CIElab, las fórmulas de diferencia de color y el uso de la norma europea en 12878 en morteros y hormigones coloreados. Ciencia y Tecnología de los Materiales. 2016; (6): 41-53. 
7. Reyes Y, Vergara I, Torres O, González EE. Contaminación por metales pesados: implicaciones en salud, ambiente y seguridad alimentaria. Revista Ingeniería, Investigación y Desarrollo. 2016; 16 (2): 66-77.

8. Martínez AG, Díaz GC, Ramírez RL. Estudio comparativo de obtención, caracterización y actividad antioxidante de quitosano a partir de exoesqueletos de camarón estero y camarón de altamar. Investigación y Desarrollo en Ciencia y Tecnología de Alimentos. 2019; 4: 1002-1013.

9. Espinoza EV. Propiedades físicas y biológicas de dos tipos de esponjas de quitosano, para su aplicación como biomaterial. [tesis de pregrado]. Lima: Universidad Nacional Mayor de San Marcos.

10. Moreno E, Alfaro R, Guzmán V, Rimaycuna J, Cruz G. Efecto de la reducción del peso molecular de quitosano obtenido de plumas de pota (Dosidicus gigas) utilizando dos métodos de despolimerización sobre su capacidad antimicrobiana. Revista de Investigación Científica Manglar. 2016; 13 (1): 25-33.

11. Morante J, Agnieszka A, Bru-Martínez R, Carranza M, Pico R, Nieto E. Distribución, localización e inhibidores de las polifenoloxidasas en frutos y vegetales usados como alimento. Ciencia y Tecnología. 2014; 7(1): 23-31.

12. Barea M. Caracterización, capacidad antioxidante y perfil fenólico de frutas subtropicales producidas y comercializadas en la costa de Granada-Málaga [tesis postgrado]. Granada: Universidad de Granada; 2015.

13. García M. Potencialidades de la quitosana como agente antioxidante y antimicrobiano en la industria alimentaria. Ciencia y Tecnología de Alimentos. 2015; 25(1): 69-76.

14. Mármol Z, Páez G, Rincón M, Araujo K, Aiello C, Chandler C, Gutiérrez E. Quitina y Quitosano polímeros amigables. Una revisión de sus aplicaciones. Revista Tecnocientífica URU. 2011; 1: 53-58.

15. Silveira A. Uso de aditivos y métodos físicos para mantener la calidad de los productos de IV gama o mínimamente procesados. Agrociencia Uruguay. 2017; 21(1): 1-6.

16. Jiang Y, Duan X, Zheng H. Browning: Enzymatic Browning. Encyclopedia of Food and Health. 2016: 508-514. doi:10.1016/b978-0-12-384947-2.00090-8

17. Chávez H, Acevedo S, Luzardo E, Valbuena A, Colina M. Obtención y caracterización de quitosano entrecruzado con ácido cítrico. Revista Iberoamericana de Polímeros y Materiales. 2018; 19 (5): 227 - 235.

18. Cob N, Tello J, Escamilla J, Vargas L, Tamayo, J. Actividad de la polifenoloxidasa en el gel de sábila (Aloe vera Mill). Revista Iberoamericana de Tecnología Poscosecha. 2010; 11 (2): 221-227.

19. Wade LG. Química orgánica. México: Pearson; 2012.

20. Ibarz A, Casero T, Miguelsanz R, Pagan J. Efecto de la temperatura en la cinética de pardeamiento no enzimático en zumos clarificados de pera con diferentes contenidos de sólidos solubles. Agroquímica y Tecnología de Alimentos. 1989; 29: 530-537. 